\title{
Hybrid method for understanding black-hole mergers: Head-on case
}

\author{
David A. Nichols* and Yanbei Chen ${ }^{\dagger}$ \\ Theoretical Astrophysics 350-17, California Institute of Technology, Pasadena, California 91125, USA
}

(Received 12 July 2010; published 8 November 2010)

\begin{abstract}
Black-hole-binary coalescence is often divided into three stages: inspiral, merger, and ringdown. The post-Newtonian (PN) approximation treats the inspiral phase, black-hole perturbation (BHP) theory describes the ringdown, and the nonlinear dynamics of space-time characterize the merger. In this paper, we introduce a hybrid method that incorporates elements of PN and BHP theories, and we apply it to the head-on collision of black holes with transverse, antiparallel spins. We compare our approximation technique with a full numerical-relativity simulation, and we find good agreement between the gravitational waveforms and the radiated energy and momentum. Our results suggest that PN and BHP theories may suffice to explain the main features of outgoing gravitational radiation for head-on mergers. This would further imply that linear perturbations to exact black-hole solutions can capture the nonlinear aspects of head-on binary-black-hole mergers accessible to observers far from the collision.
\end{abstract}

DOI: $10.1103 /$ PhysRevD.82.104020

PACS numbers: 04.25.Nx, 04.30.- w, 04.70.- s

\section{INTRODUCTION}

Even prior to the complete numerical-relativity simulations of black-hole-binary mergers [1-4], black-hole collisions were thought to take place in three stages: inspiral (or infall), merger, and ringdown. During inspiral, the speed of the holes is sufficiently low and the separation of the bodies is large enough that the system behaves like two separate particles that follow the post-Newtonian (PN) equations of motion. Eventually, the black holes become sufficiently close that the dynamics given by the PN expansion significantly differ from those of full relativity. This stage is the merger, during which the two black holes become a single, highly distorted, black hole. The merger phase is brief; the strong deformations lose their energy to gravitational radiation, and the system settles down to a weakly perturbed, single black hole. The ringdown phase describes these last small oscillations of the black hole.

Of the three stages of binary-black-hole coalescence, merger remains the most inaccessible to analytical tools. Nevertheless, full numerical relativity is not the only technique to have success investigating merger. Historically, most analytical investigations of the merger phase arise from trying to extend the validity of perturbative techniques, particularly black-hole-perturbation (BHP) and PN theories. Those researchers working from a BHP approach try to push the approximation to hold at earlier times, whereas those employing a PN method attempt to stretch the technique to hold later into merger. Alternatively, one can see if there are exact, nonlinear analytical models whose dynamics can represent various aspects of black-hole-binary mergers. Rezzolla, Macedo, and Jaramillo [5] recently took this latter approach in their study of antikicks from black-hole mergers. In their work,

\footnotetext{
*davidn@tapir.caltech.edu

†yanbei@tapir.caltech.edu
}

they showed that they could relate the curvature anisotropy on the past apparent horizon of a Robinson-Trautman space-time to the kick velocity (computed from the Bondi momentum). Through appropriate tuning of the initial data, they could recover kick velocities found in numerical-relativity simulations of unequal-mass, insprialing black holes. While this type of approach is interesting and has proved successful, our work focuses on using perturbative approaches (and we will, therefore, take a more comprehensive look at the prior use of perturbative methods to understand mergers).

From the BHP side, Price and Pullin [6], initially, and many collaborators, subsequently, (see, e.g., [7]) developed the "close-limit approximation" (CLA). This technique begins with initial data containing two black holes that satisfy the vacuum Einstein equations and splits it into a piece representing the final, merged black hole and perturbations upon that black hole. The exact form of the initial data varies in the CLA, but for head-on collisions of black holes, it typically involves some variation of Misner [8], Lindquist [9], or Brill-Lindquist [10] time-symmetric, analytic, wormholelike solutions. To investigate the late stages of an inspiral, the CLA usually begins from nontime-symmetric, but conformally flat, multiple black-hole initial data set forth by Bowen and York [11]. Independent of the initial data, however, the CLA translates the original problem of merger into a calculation involving BHP theory. The CLA does not allow for a very large separation of the black holes; as a result, only the very end of the merger is captured in this process. Moreover, "junk radiation" appears in the waveform because the initial data do not describe the binary black-hole-merger space-time in both the wave zone and the near zone. Unlike in full numericalrelativity simulations where the junk radiation leaves the grid during the well understood inspiral phase, in the CLA the junk radiation appears during the merger stage. This radiation (both from the absence of waves in the initial data 
and from errors in the near-zone physics), therefore, is difficult to disentangle from the physical waveform.

The Lazarus Project (see, e.g., [12]) followed roughly the same approach as the CLA, but it used even more realistic black-hole-binary initial data for its CLA calculation; namely, its initial data came from a numericalrelativity simulation just prior to merger. At the same time, however, because the initial data is now numerical, one loses the analytical understanding of the properties of space-time near merger. More recently, Sopuerta, Yunes, and Laguna [13] applied the CLA in combination with PN flux formulas to obtain an estimate of the gravitational recoil from unequal-mass binaries (including binaries with small eccentricity [14]). They proposed using more realistic initial data in the CLA, which Le Tiec and Blanchet [15] ultimately carried out. Le Tiec and Blanchet chose to use the 2PN metric (keeping only the first post-Minkowski terms) as initial data for the CLA, and they applied it with considerable success to inspirals of unequal-mass black-hole binaries in a paper with Will [16]. Despite the improved initial data, this approach does not eliminate the problem of junk radiation discussed above. It would be of interest to see if even more realistic initial data, such as that of Johnson-McDaniel, Yunes, Tichy, and Owen [17] would lead to improved results within the CLA.

From the PN side, Buonanno et al., [18], as well as Damour and Nagar [19] take a different approach to understanding the physics of merger. Using the effective-onebody (EOB) method [20], they study the dynamics of the system until roughly the beginning of the merger phase. To obtain a complete waveform, they attach a ringdown waveform by smoothly fitting quasinormal modes to the EOB inspiral and plunge waveform. When they calibrate the two free parameters of this model to numerical-relativity waveforms, the EOB results match numerical-relativity waveforms precisely. In this method, they fit the PN dynamics and the ringdown at the light ring of the EOB particle motion; it is not immediately apparent, however, what this feature tells about the nature of merger.

As a result, there remains a need to develop simple analytical models that help reveal the behavior of spacetime during merger. Toward this end, it is helpful to delve deeper into the question of what exactly is the merger. First, the inspiral-merger-ringdown classification is based on the validity of the PN expansion and that of BHP. The inspiral, in other words, is just the set of times for which the PN expansion holds on the whole spatial domain (to a given level of accuracy). Correspondingly, the ringdown is the times for which BHP works everywhere throughout space. Merger, in this picture, is just the gap between those times during which PN and BHP theories give accurate results.

In this paper, we propose that we can push each approximation technique beyond its current range of use, as long as we do not apply it to all of space at a given time. We observe that at any time, there is a region outside a certain radius from the center of mass in which BHP applies. While this seemingly runs contrary to the common notion that PN theory is the natural description of the weak-field region of a black-hole-binary space-time, a black-hole metric in the limit of radii much larger than the mass and binary separation is identical to that of PN in the same region. If the PN expansion applies to the remaining portion of the space-time (within the region where BHP holds), then BHP and PN theories could cover the entire physics of black-hole-binary coalescence. While it is somewhat unreasonable to suppose that PN theory truly applies to the strong-field region of a binary-black-hole space-time, revisiting Price's treatment of nonspherical stellar collapse suggests that this may not be essential.

In Price's 1972 paper [21], he addresses, among other issues, why aspherical portions of stellar collapse quickly disappear when, in fact, one could plausibly argue the contrary. Namely, if any nonspherical perturbations asymptote to the horizon (from the perspective of an observer far away), they would remain visible to this observer indefinitely. Price realizes, however, that the exterior of a collapsing star is just the Schwarzschild solution (due to Birkhoff's theorem, up to small perturbations), and perturbations on the Schwarzschild space-time evolve via a radial wave equation in an effective potential. Moreover, he notes that when the surface of the star passes through the curvature effective potential of the Schwarzschild spacetime, the gravitational perturbations induced by the star redshift. Finally, because the effective potential reflects low-frequency perturbations, the space-time distortions produced by the stellar interior become less important, and observers outside the star ultimately see it settle into a spherical black hole in a finite amount of time. Most importantly, this argument does not depend strongly upon the physics of the stellar interior; as long as there is gravitational collapse to a black hole, this idea holds.

In this paper, we adopt this idea, but we replace the stellar physics of the interior with a PN, black-hole-binary space-time (see Fig. 1). While in Price's case, the division of space-time into two regions comes naturally from tracking the regions of space containing the star and vacuum, in our case the split is somewhat more arbitrary; one simply needs to find a region in which both PN and BHP theories hold, to some level of accuracy. How we choose the boundary between the two regions and the quantities that we evolve are topics that will be discussed in greater detail in Sec. II.

To test the above idea in this paper, we study a head-on collision of two black holes with transverse, anti-aligned spins and compare the waveforms and energy-momentum flux obtained from our approximation method with the equivalent quantities from full numerical simulations. Specifically, we organize this paper as follows. In Sec. II, we give a more detailed motivation for our model, and we 


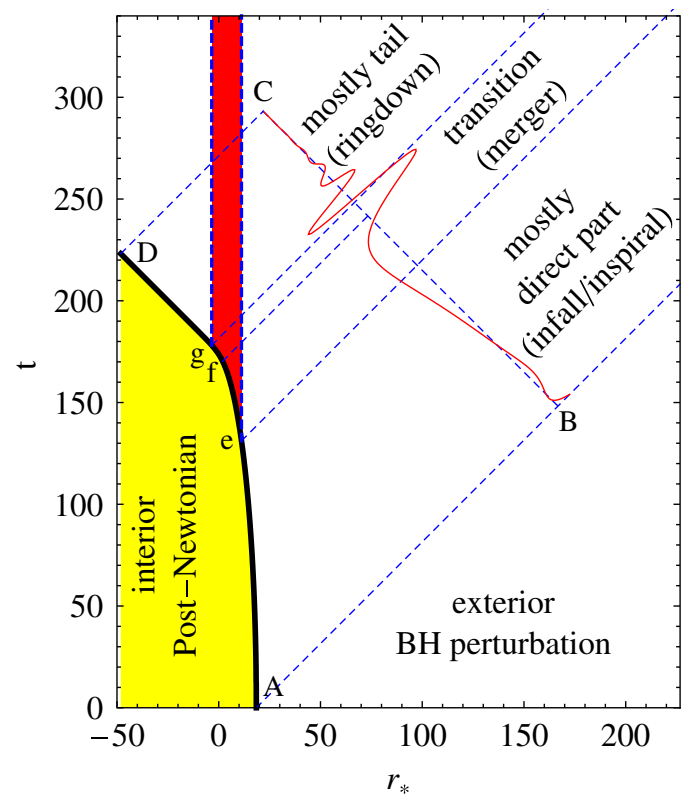

FIG. 1 (color online). This figure depicts a space-time diagram of a black-hole collision, modeled after Price's description of stellar collapse. We choose the trajectory of the two holes as a way to separate the space-time into an interior and an exterior region. The exterior region is a perturbed, black-hole space-time, whereas the interior is that of a post-Newtonian (PN) black-holebinary system [shaded in yellow (light gray)]. The red (dark gray) region of the diagram shows the place at which the effective potential of the black-hole is significantly greater than zero. This formalism allows us to divide the waveform into three sections: inspiral (or infall), which extends from the beginning of the binary's evolution until when the $l=2$ effective potential of the exterior BHP space-time starts to be exposed; merger, which extends from the end of inspiral to when the majority of the exterior potential is revealed; and ringdown, which represents the remainder. We overlay the even-parity, $(l=2, m=0)$ mode of the waveform.

then present the mathematical procedure we use in our method, for an equal-mass, head-on collision of black holes. In Sec. III, we present an explicit calculation for the head-on collision of two black holes with transverse, anti-aligned spins, and we compare waveforms, radiated energy, and radiated linear momentum, from our model with the equivalent quantities from a full numericalrelativity simulation. In Sec. IV we discuss how our method can help interpret the waveform during merger, and finally, in Sec. V, we conclude. We will use geometrical units $(G=c=1)$ and Einstein summation convention throughout this paper.

\section{A DETAILED DESCRIPTION OF THE METHOD}

\section{A. Further motivation}

Before going into the details of our procedure, it is worth spending some time discussing why our specific implementation of PN and BHP theories will help avoid some of the difficulties that arose in other methods in the Introduction and noting the limitations and assumptions that underlie our approach.

It is certainly hard to argue that existing orders of PN (up to $v^{6}$ in the metric, for near-zone dynamics [22]) and BHP (up to second order for Schwarzschild, see [23] for a gauge-invariant formulation) theories are accurate in the whole space, simultaneously. Nevertheless, it is plausible to argue that these approximation techniques cover different spatial regions at different times in a way such that each theory is either valid to a reasonable level of accuracy or occupying a portion of space-time that will not influence physical observables where it fails. Using an approach of this type, we aim to get the most out of the approximation methods.

Specifically, we find that the following procedure gives good agreement with the waveform of a numericalrelativity simulation presented in Sec. III. First, we have the reduced mass of the binary follow a plunging geodesic in the Schwarzschild space-time. Then, we divide this trajectory in half to make a coordinate radius (and thus a coordinate sphere) that passes through the centers of the black holes. The set of all the coordinate spheres defines a timelike surface in space-time. Finally, we apply PN theory within this timelike surface and BHP on the exterior. The two theories must agree on this timelike surface, which we will subsequently call the shell.

Matching PN and BHP theories on this shell has certain advantages. Because BHP theory relies upon a multipole expansion, this makes it necessary to treat the PN interior in terms of multipoles of the potentials. For one, this is useful, because physical observables like the radiated energy and momentum very often do not need many multipoles to find accurate results. (In fact, in our example in Sec. III, we see that the quadrupole perturbations alone suffice.) Second, a multipole expansion may also be helpful for the convergence of the approach. For two point particles, for example, each multipole component of the Newtonian potential $U_{N}^{(l)}$ at the location of the particles satisfies $U_{N}^{(l)} \lesssim M / R$, where $M$ is the mass of the binary and $R$ is the distance from the center of mass. This is small for much of the infall, when $R \gg M$, and even when the binary reaches what will be the peak of the effective potential of the merged black hole, $U_{N}^{(l)} \sim 1 / 3$.

Because the effective potential of the final black hole tends to mask perturbations within (as they are redshifted and cannot escape), our hope (supported by the example in Sec. III) is that PN theory is still reasonably accurate at the peak of the potential. Then, in our approach, the PN error will be hidden by this potential and, along with BHP in the exterior, it will suffice to explain the physics outside the black hole (in particular the gravitational waveform and the energy and momentum flux). Of course whether such a mechanism exists is not easily deduced analytically from first principles; only by comparing our results with those 
from full numerical relativity will we test this premise (which we do in Sec. III B).

If this holds, our procedure has several advantages. For one, the matching works well at larger separations (which diminishes the influence of junk radiation). More importantly, though, as the matching shell moves from large separations at early times to the vicinity of the horizon at late times, the space-time smoothly transitions from predominantly PN to essentially BHP. One can see this most clearly in the way that the effective potential is revealed in Fig. 1. This leads to a waveform that smoothly transitions between an inspiral at early times to a ringdown at late times. Whether we correctly capture the merger phase is most easily confirmed by comparing with results from numerical relativity. Finally, our method can give a way to interpret the waveform during the different stages of a binary-black-hole coalescence by relating parts of the waveform with the retarded position of the matching shell at that point (for further detail, see Sec. IV).

There is also reason to suspect that the domain of BHP may extend to relatively early times in the merger, outside the binary. A paper by Racine, Buonanno, and Kidder [24] gives evidence in favor of this idea. In particular, they find, when studying the "superkick" configuration (an equal-mass, circular binary of black holes with transverse, antiparallel spins), that the higher-order spin-orbit contributions to linear-momentum flux dominate over the leading-order terms. These terms include both direct higher PN terms and tail terms, where the tail refers to gravitational radiation that is scattered off of space-time curvature and propagates within the light cone (as opposed to the direct piece that propagates on the light cone). This suggests that even early on, the background curvature plays an important role in generating the kick. Whether a Schwarzschild black hole properly represents this curvature is another idea that is difficult to argue for directly, but can be confirmed by comparison with the results of numerical relativity.

In its current implementation, the feature of our approach that is most arbitrary is setting the boundary between the PN interior and the BHP exterior. Nevertheless, our procedure works for head-on collisions, and in future work, we will develop a more systematic way of treating the boundary.

\section{B. Details of the implementation}

The procedure that we follow can be broken down, more or less, into five steps: (i) we describe relevant physics in the PN interior; (ii) we match the metric of the PN interior to the BHP metric through a boundary; (iii) we explicitly construct the boundary between the PN and BHP space-times; (iv) we evolve the metric perturbations in the exterior Schwarzschild space-time; (v) we extract the waveforms and compute the radiated energy and momentum. We shall devote a subsection to each of these topics below.
Before we do so, however, it will be helpful to clarify our notation regarding the different coordinates we use for the two metrics and the matching shell. In the PN coordinate system, we use Minkowski coordinates $(T, X, Y, Z)$ and spherical-polar coordinates $(R, \Theta, \Phi)$ within spatial slices. We will only consider linear perturbations to Minkowski in the harmonic gauge. For our BHP, we employ Schwarzschild coordinates $(t, r, \theta, \varphi)$, and similarly, we only examine linear perturbations to the Schwarzschild space-time. As we will show in Sec. II B 3, we can match these descriptions when $R, r \gg M$. This procedure is accurate up to terms of order $(M / R)^{2}$ in the monopole part of the metric and $M / R$ in the higher-multipole portions, assuming that we relate the two coordinate systems by

$$
T=t, \quad \Theta=\theta, \quad \Phi=\varphi, \quad R=r-M .
$$

The identification above allows us to label every point in space-time by two sets of coordinates, $(t, r, \theta, \varphi)$ and $(t, R, \theta, \varphi)$, where $R=r-M$. Because our program relies upon applying PN theory in an interior region and BHP on the exterior, it is therefore natural to talk about a coordinate shell at which we switch between PN and BHP descriptions of the space-time. On this shell, we can use either the Minkowski or Schwarzschild coordinates.

In keeping with the notation above, we shall denote the separation of the binary by $A(t)=2 R(t)$ in PN coordinates and $a(t)=2 r(t)$ in Schwarzschild coordinates. Finally, we will denote the radial coordinate on the boundary by adding a subscript $s$ to the PN or Schwarzschild coordinate radii [e.g., $R_{s}(t)=A(t) / 2$ or $r_{s}(t)=a(t) / 2$ ]. We summarize the two coordinate systems and how they match in Table I and Fig. 2.

While we introduce a new method of matching PN and BHP theories, the idea of combining PN and BHP approximations is not new. In fact, it is at the core of the effective-one-body formalism of Buonanno and Damour [20]. In the EOB description, however, they match the point-particle Hamiltonians of PN and BHP theories, rather than joining the space-time geometry. It would be interesting, as a future study, to see whether one can combine our procedure with that of the EOB to produce a geometrical EOB approach. Le Tiec and Blanchet [15], on the other hand performed a more accurate matching between $\mathrm{PN}$ and BHP in their close-limit calculation with 2PN initial data, but their matching only takes place on a single spatial slice of initial data. It would also be interesting to extend their higher-order approach to our procedure as well.

\section{The PN interior solution}

For our method, we will need to describe the metric of the PN space-time in the interior, which we do at leading Newtonian order: 
TABLE I. This table summarizes our notation for the coordinates, the binary separation, and the matching radius that we use for the PN space-time, the BHP space-time, and the matching region between the two.

\begin{tabular}{lccc}
\hline \hline & PN Space-time & Matching Shell & Perturbed Schwarzschild Space-time \\
\hline Coordinates & $(t, R, \theta, \varphi)$ & $\left(t, R_{s}(t), \theta, \varphi\right)$ or $\left(t, r_{s}(t), \theta, \varphi\right)$ & $(t, r, \theta, \varphi), r=R+M$ \\
Binary Separation & $A(t)$ & $A(t)$ or $a(t)$ & $a(t)$ \\
Matching Radius & $R(t)=A(t) / 2$ & $R_{s}(t)=a(t) / 2-M$ or $r_{s}(t)=A(t) / 2+M$ & $r(t)=a(t) / 2$ \\
\hline \hline
\end{tabular}

$$
\begin{aligned}
d s_{\mathrm{PN}}^{2}= & -\left(1-2 U_{N}\right) d t^{2}-8 w_{i} d t d X^{i} \\
& +\left(1+2 U_{N}\right) \delta_{i j} d X^{i} d X^{j} .
\end{aligned}
$$

Here $U_{N}$ is the Newtonian potential and $w_{i}$ is the gravitomagnetic potential, and the index $i$ runs over $X, Y$, and $Z$. Our notation follows [25] [the above takes the results of Eq. (2.1) of that paper]. We then expand the Newtonian potential, $U_{N}$, into multipoles, keeping only the lowest multipoles necessary to complete the calculation. In this paper, the monopole and quadrupole pieces suffice (the dipole piece can always be made to vanish),

$$
U_{N} \approx U_{N}^{(0)}+U_{N}^{(2)}
$$

The quadrupole piece can be expressed as a term without angular dependence times a spherical harmonic, as is done below,

$$
U_{N}^{(2)}=U_{N}^{2, m} Y_{2, m}(\theta, \varphi)
$$

We shall follow the same procedure with the gravitomagnetic potential, although here we will, temporarily, keep the dipole term,

$$
w_{i} \approx w_{i}^{(1)}+w_{i}^{(2)} \text {. }
$$

We will be able to remove the dipole term through gauge transformations, but this discussion is much simpler on a case-by-case basis. When we write the gravitomagnetic potential, w, in spherical-polar coordinates, we will be able to remove the radial component through a gauge transformation. We will, therefore, consider just the $\theta$ and $\varphi$ components of $\mathbf{w}$, and when writing it in index notation, we will denote them with Latin letters from the beginning of the alphabet (e.g., $a, b=\theta, \varphi$ ). We can then expand the components $w_{a}^{(2)}$ in terms of two vector spherical harmonics,

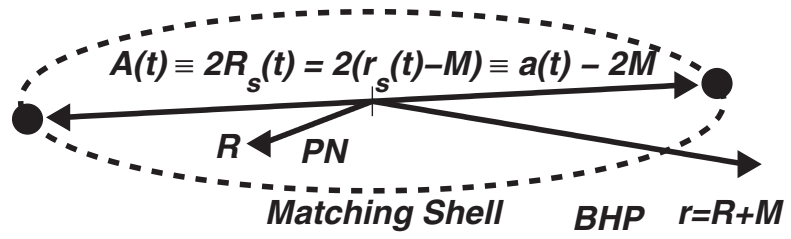

FIG. 2. This figure shows, at a given moment in time, the Schwarzschild and PN radial coordinates, the binary separation, and the position of the shell where we match the two theories.

$$
w_{a}^{(2)}=w_{(\mathrm{e})}^{2, m} \nabla_{a} Y_{2, m}(\theta, \varphi)+w_{(\mathrm{o})}^{2, m} \epsilon_{a}^{b} \nabla_{b} Y_{2, m}(\theta, \varphi) .
$$

Here $\nabla_{a}$ is the covariant derivative on a 2 -sphere, and $\epsilon_{a}{ }^{b}$ is the Levi-Civita tensor (with nonzero components $\epsilon_{\theta}{ }^{\varphi}=1 / \sin \theta$ and $\left.\epsilon_{\varphi}{ }^{\theta}=-\sin \theta\right)$. A convenient abbreviation for the two spherical harmonics above is

$$
w_{a}^{(2)}=w_{(\mathrm{e})}^{2, m} Y_{a}^{2, m}+w_{(\mathrm{o})}^{2, m} X_{a}^{2, m} .
$$

The two terms are denoted by (e) and (o) as a shorthand for even and odd, because they transform as $(-1)^{l}$ and $(-1)^{l+1}$ under parity transformations, respectively. The odd- and even-parity vector spherical harmonics are given explicitly by

$$
X_{\theta}^{l, m}=-\frac{1}{\sin \theta} \frac{\partial Y^{l m}}{\partial \varphi}, \quad X_{\varphi}^{l, m}=\sin \theta \frac{\partial Y^{l m}}{\partial \theta}
$$

and

$$
Y_{\theta}^{l, m}=\frac{\partial Y^{l m}}{\partial \theta}, \quad Y_{\varphi}^{l, m}=\frac{\partial Y^{l m}}{\partial \varphi},
$$

respectively. These are the only parts of the PN metric that will be necessary for our approach.

\section{Matching to perturbed Schwarzschild}

We then note that the Schwarzschild metric takes the form

$$
d s^{2}=-\left(1-\frac{2 M}{r}\right) d t^{2}+\left(1-\frac{2 M}{r}\right)^{-1} d r^{2}+r^{2} d^{2} \Omega,
$$

where the last piece is the metric of a 2-sphere. We will use $r$ without any subscript to denote the Schwarzschild radial coordinate. When $M \ll r$ the Schwarzschild metric takes the form

$$
d s^{2} \approx-\left(1-\frac{2 M}{r}\right) d t^{2}+\left(1+\frac{2 M}{r}\right) d r^{2}+r^{2} d^{2} \Omega .
$$

By making the coordinate transformation $R=r-M$, and identifying $M / R$ with the monopole piece of the Newtonian potential, $U_{N}^{(0)}$, then one can find that the Schwarzschild metric takes the form of the Newtonian metric in spherical coordinates, 


$$
d s^{2} \approx-\left(1-2 U_{N}^{(0)}\right) d t^{2}+\left(1+2 U_{N}^{(0)}\right)\left(d R^{2}+R^{2} d^{2} \Omega\right) .
$$

This similarity between the PN and Schwarzschild metrics suggests a way to match the two at the boundary. We will assume that the monopole piece of the Newtonian potential becomes the $M / r$ term in the Schwarzschild metric. For the remaining pieces of the Newtonian metric (namely $U_{N}^{(2)}$ and $w_{i}^{(2)}$ ) we will translate them directly into the Schwarzschild metric after performing any needed gauge transformations to make such a direct match reasonable.

The original works on perturbations of the Schwarzschild space-time are those of Regge and Wheeler [26] for the odd-parity perturbations and Zerilli [27] for the even-parity perturbations. Moncrief [28] then used a variational principle to show that one can derive quantities from the metric perturbations of Regge, Wheeler, and Zerilli that satisfy a well-posed, initialvalue problem in any coordinates that deviate from Schwarzschild at linear order in perturbation theory. These quantities are related to the gravitational waves at infinity, and they satisfy a one-dimensional wave equation in a potential. We follow Moncrief's approach in computing these so-called gauge-invariant metric perturbation functions, but for our notation, we use that of a recent review article by Ruiz et al. [29].

Both the even-parity [transform as $(-1)^{l}$ under parity] and odd-parity [transform as $(-1)^{l+1}$ under parity] perturbations are not very difficult to find. By writing the PN metric in spherical-polar coordinates,

$$
\begin{aligned}
d s^{2}= & -\left(1-2 U_{N}^{(0)}-2 U_{N}^{(2)}\right) d t^{2}+\left(1+2 U_{N}^{(0)}+2 U_{N}^{(2)}\right) \\
& \times\left(d R^{2}+R^{2} d^{2} \Omega\right)-8 w_{b}^{(2)} d t d x^{b},
\end{aligned}
$$

where $d x^{b}=d \theta, d \varphi$, one can see that the even-parity perturbations are nearly diagonal in the metric. In fact, at leading Newtonian order, it is exactly diagonal, because the nondiagonal term coming from $w_{(\mathrm{e})}^{2, m}$ arises at a higher PN order. We will show this explicitly in Sec. III. For this reason, we only consider the diagonal metric components in the discussion below.

The even-parity metric perturbations in Schwarzschild are often denoted

$$
\begin{array}{ll}
\left(h_{t t}^{l, m}\right)_{(\mathrm{e})}=H_{t t}^{l, m} Y^{l, m}, & \left(h_{r r}^{l, m}\right)_{(\mathrm{e})}=H_{r r}^{l, m} Y^{l, m}, \\
\left(h_{\theta \theta}^{l, m}\right)_{(\mathrm{e})}=r^{2} K^{l, m} Y^{l, m}, & \left(h_{\varphi \varphi}^{l, m}\right)_{(\mathrm{e})}=r^{2} \sin ^{2} \theta K^{l, m} Y^{l, m},
\end{array}
$$

a specialization of Eqs. (57)-(59) of Ruiz et al. Thus, by matching the two metrics, one can see that

$$
H_{t t}^{2, m}=H_{r r}^{2, m}=K^{2, m}=2 U_{N}^{2, m} .
$$

The odd-parity term is somewhat simpler, because there is only one metric perturbation in Schwarzschild to match at leading order,

$$
\left(h_{t \theta}^{l, m}\right)_{(\mathrm{o})}=h_{t}^{l, m} X_{\theta}^{l, m}, \quad\left(h_{t \varphi}^{l, m}\right)_{(\mathrm{o})}=h_{t}^{l, m} X_{\varphi}^{l, m},
$$

Eq. (61) of Ruiz et al. [29]. From this, one can find that

$$
h_{t}^{2, m}=-4 w_{(\mathrm{o})}^{2, m} \text {. }
$$

The matching procedure thus gives a way to produce perturbations in the Schwarzschild space-time.

\section{The boundary shell}

We now must find a boundary region where one can match a PN metric expanded in multipoles with a perturbed Schwarzschild metric. For head-on collisions, we find that the boundary can be a spherical shell whose radius varies in time as the binary evolves. We can motivate where this boundary should be with a few simple arguments, but the true test of the matching idea will come from comparisons with exact waveforms from numerical relativity.

We know that at early times and for larger separations of the black holes, the PN space-time is valid around the two holes; thus it is not unreasonable to suppose that the shell should have a radius equal to half the binary separation. Later in the evolution, BHP will be valid everywhere, so the shell should asymptote to the horizon of the merged hole (as seen by outside observers). The trajectory of the shell should be smooth throughout the entire process, as well. Finally, the boundary should mimic the reduced-mass motion of the system, which physically generates the gravitational waves.

A simple way to achieve this quantitatively is instead of having the motion of the reduced mass follow the PN equations of motion, we impose that it undergoes plunging geodesic motion in the Schwarzschild space-time. Given that the exterior space-time is a perturbed Schwarzschild, and that we are matching the two approximations on a shell that passes through the centers of the two black holes, it is just as reasonable to use a trajectory in the Schwarzschild space-time. Moreover, at large separations, the motion of the reduced mass of the system in both Schwarzschild and PN are quite similar; we primarily choose the geodesic in Schwarzschild for its behavior at late times. For completeness, we write down the differential equation we use to find the motion of a radial geodesic in Schwarzschild. Since we think of the black-holes as point particles residing in the $\mathrm{PN}$ coordinate system, we write the evolution of the binary separation $A(t)$, measured in the PN coordinates,

$$
\begin{aligned}
& \frac{d A(t)}{d \tau}=-\sqrt{B^{2}-(1-2 M / A(t))}, \\
& \frac{d t}{d \tau}=B\left(1-\frac{2 M}{A(t)}\right)^{-1}
\end{aligned}
$$

where $B$ is a positive constant $[B=1-2 M / A(0)$ for a head-on plunge from rest]. This expression can be found in many sources; see, for example [30]. The coordinate radius of the shell in the PN space-time is just half the distance $A(t)$, 


$$
R_{s}(t)=\frac{1}{2} A(t) .
$$

Since the PN and Schwarzschild radii are related by $R=$ $r-M$, one can find that in the Schwarzschild coordinates, the position of the shell is given by

$$
r_{s}(t)=\frac{1}{2} A(t)+M
$$

In the Schwarzschild coordinates, since $A(t)$ goes to $2 M$ at late times, the radius of the shell asymptotes to the horizon. More specifically, let us first define $\delta r_{s}(t)=r_{s}(t)-2 M$ and then note that at late times the trajectory approaches the speed of light as it falls toward the horizon. In terms of the tortoise coordinate, $r_{*}=r+2 M \log [r /(2 M)-1]$, this occurs when $v=t+r_{*}=$ const. Writing this with respect to the variable $\delta r_{s}(t)$, we find

$$
-\frac{t}{2 M} \sim \frac{\delta r_{s}(t)}{2 M}+\log \left(\frac{\delta r_{s}(t)}{2 M}\right) .
$$

We neglected the constant value of $v$ because doing so has no affect on finding the scaling of $r_{s}(t)$ at late times $t$. The equation above has a solution in terms of the Lambert $W$ function, $W(x)$, given by

$$
\delta r_{s}(t) \sim W\left(e^{-t /(2 M)}\right) .
$$

Because we are interested in the behavior at large $t$, $e^{-t /(2 M)}$ is small, and we can use the leading-order term in the Taylor series for the Lambert $W$ function, $W(x) \sim x+O\left(x^{2}\right)$. We find that

$$
\delta r_{s}(t) \sim e^{-t /(2 M)} .
$$

Thus, matching the space-times at half the PN separation of the binary and having the separation of the binary evolve via a Schwarzschild geodesic in the PN coordinate system makes the shell track the PN reduced-mass motion at early times, but still head to the horizon at late times. We illustrate these different behaviors by plotting the full trajectory of the shell $r_{s}(t)$, the trajectory of a shell that follows a plunging Newtonian orbit [which we denote by $r_{s,(N)}(t)$ and which represents the behavior of the shell at early times], and $e^{-t /(2 M)}$ (the late-time behavior of the shell) in Fig. 3. We choose the initial values of the trajectory to conform with those of the numerical simulation with which we compare in Sec. III B. The upper and lower insets show how the shell's trajectory converges to the Newtonian behavior (at early times) and the expected exponential decay at late times.

While choosing the trajectory of the shell might have a slightly ad hoc feel, in future work we will develop a framework that determines the shell motion consistently through radiation reaction.

\section{Black-hole perturbations}

One can then take the odd- and even-parity metric perturbations from the second subsection and transform them into two quantities, the Regge-Wheeler and Zerilli

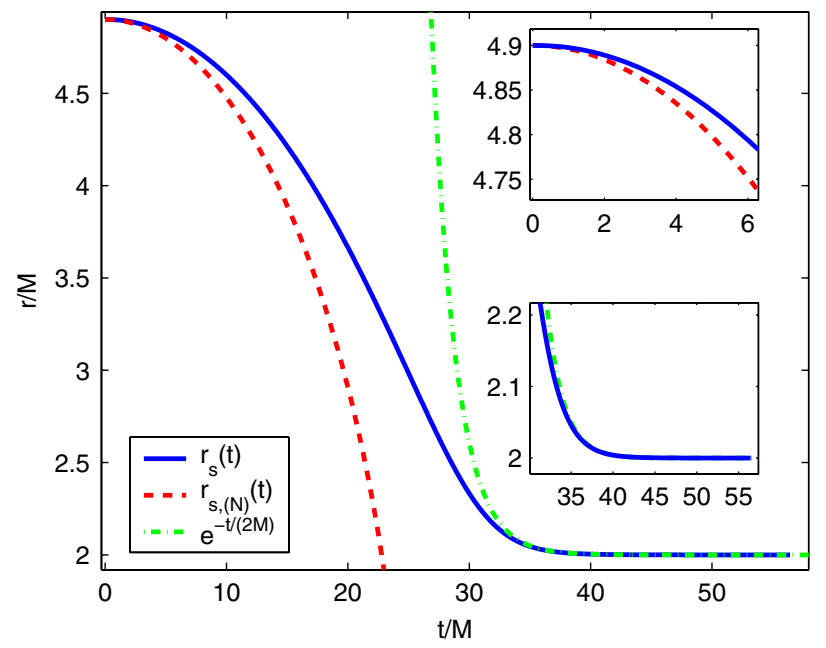

FIG. 3 (color online). This figure shows the trajectory of the boundary shell as the solid blue (black) curve labeled by $r_{s}(t)$. The other two curves show the early- and late-time behavior of the shell. The red (gray) dashed curve labeled by $r_{s,(N)}(t)$ shows the trajectory of a shell that follows the Newtonian equations of motion for a plunge from rest. The green (light gray) dashed and dotted curve [denoted by $e^{-t /(2 M)}$ ] shows the exponential convergence to the horizon at the rate expected in a Schwarzschild space-time. The upper inset shows how the shell agrees with a Newtonian plunge from rest at early times, and the lower inset shows how the shell converges exponentially to the horizon at the expected rate.

functions, respectively, that each satisfy a simple wave equation. We first treat the even-parity perturbations. Equations (63)-(65) of Ruiz et al. [29] show how to take metric perturbations and transform them into the evenparity, gauge-invariant Zerilli function. Substituting our Eq. (15) into those three of Ruiz, we find

$$
\begin{aligned}
\Psi_{(\mathrm{e})}^{2, m}= & \frac{2 r}{3}\left\{U_{N}^{2, m}+\frac{r-2 M}{2 r+3 M}\left[\left(1-\frac{2 M}{r}\right) U_{N}^{2, m}\right.\right. \\
& \left.\left.-r \partial_{r} U_{N}^{2, m}\right]\right\},
\end{aligned}
$$

where $\partial_{r}$ is just the radial derivative with respect to the Schwarzschild radial variable. The odd-parity perturbations come directly from applying our Eq. (17) to Eq. (67) of Ruiz et al. [29]. This gives that

$$
\Psi_{(\mathrm{o})}^{2, m}=2 r\left(\partial_{r} w_{(\mathrm{o})}^{2, m}-\frac{2}{r} w_{(\mathrm{o})}^{2, m}\right) \text {. }
$$

The odd- and even-parity perturbations then evolve according to the Regge-Wheeler and Zerilli equations, respectively,

$$
\left(\partial_{t}^{2}-\partial_{r_{*}}^{2}+V_{(\mathrm{e}, \mathrm{o})}^{l}(r)\right) \Psi_{(\mathrm{e}, \mathrm{o})}^{l, m}=0,
$$

where $r_{*}=r+2 M \log [r /(2 M)-1]$ is the tortoise coordinate. The potentials can be expressed most concisely via the expression 


$$
V_{(\mathrm{e}, \mathrm{o})}^{l}(r)=\left(1-\frac{2 M}{r}\right)\left(\frac{\lambda}{r^{2}}-\frac{6 M}{r^{3}} U_{(\mathrm{e}, \mathrm{o})}^{l}(r)\right),
$$

where $\lambda=l(l+1)$ and

$$
U_{(\mathrm{o})}^{l}(r)=1, \quad U_{(\mathrm{e})}^{l}(r)=\frac{\Lambda(\Lambda+2) r^{2}+3 M(r-M)}{(\Lambda r+3 M)^{2}} .
$$

Here $\Lambda=(l-1)(l+2) / 2=\lambda / 2-1$. These expressions follow Eqs. (5.3)-(5.6) of [15].

In our procedure, we find it easiest to evolve the ReggeWheeler-Zerilli equations using a characteristic method. To do so, we define $u=t-r_{*}$ and $v=t+r_{*}$ and see that the evolution equation becomes

$$
\frac{\partial^{2} \Psi_{(\mathrm{e}, \mathrm{o})}^{l, m}}{\partial u \partial v}+\frac{V_{(\mathrm{e}, \mathrm{o})}^{l} \Psi_{(\mathrm{e}, \mathrm{o})}^{l, m}}{4}=0 .
$$

We will now discuss how we evolve our Regge-WheelerZerilli functions with the aid of Fig. 1.

We must provide data in two places, the matching shell (in Fig. 1 it is the lower-left curve labeled by the points A, $\mathrm{e}, \mathrm{f}, \mathrm{g}, \mathrm{D}$ ) and the initial outgoing characteristic (the line labeled by $\mathrm{AB}$ on the lower right). Once we do this, however, we can determine the Regge-Wheeler-Zerilli functions within the quadrilateral (with the one curved side) A, B, C, D. We already discussed how we determine the shell in Sec. IIB 3, and the data we provide along that curve are just the Regge-Wheeler [Eq. (25)] or Zerilli [Eq. (24)] functions restricted to that curve. The data we must provide along $\mathrm{AB}$ are less well determined. If our computational grid extended to past null infinity, then we could impose a no ingoing wave condition. At finite times, we can still impose this boundary condition, but it leads to a small spurious pulse of gravitational radiation at the beginning of our evolution. To limit the effects of this, we keep the shell at rest until the junk radiation dissipates, and then we begin our evolution. At this point, the data along the line $\mathrm{AB}$ more closely represent those of a binary about to begin its plunge.

With these data, we can then evolve the Regge-WheelerZerilli equations numerically, using the second-orderaccurate, characteristic method described by Gundlach et al. in [31]. The essence of this method is that one can use the data at a point plus those at one step ahead in $u$ and $v$, respectively, to get the next point advanced by a step ahead in both $u$ and $v$. Explicitly, if one defines $\Psi_{N}=$ $\Psi_{(\mathrm{e}, \mathrm{o})}^{l, m}(u+\Delta u, v+\Delta v), \Psi_{W}=\Psi_{(\mathrm{e}, \mathrm{o})}^{l, m}(u+\Delta u, v), \Psi_{E}=$ $\Psi_{(\mathrm{e}, \mathrm{o})}^{l, m}(u, v+\Delta v)$, and $\Psi_{S}=\Psi_{(\mathrm{e}, \mathrm{o})}^{l, m}(u, v)$, then one has that

$$
\begin{aligned}
\Psi_{N}= & \Psi_{E}+\Psi_{W}-\Psi_{S}-\frac{\Delta u \Delta v}{8} V_{(\mathrm{e}, \mathrm{o})}^{l}\left(r_{c}\right)\left(\Psi_{E}+\Psi_{W}\right) \\
& +O\left(\Delta u^{2} \Delta v, \Delta u \Delta v^{2}\right),
\end{aligned}
$$

where $r_{c}$ is the value of $r$ at the center of the discretized grid. Because our boundary data do not always lie on one of the grid points in the $(u, v)$ plane, we must interpolate the bottom point $\Psi_{(\mathrm{e}, \mathrm{o})}^{l, m}\left(u, v^{\prime}\right)$ to fall at the same value of $v$ as the next boundary point at $\Psi_{(\mathrm{e}, \mathrm{o})}^{l, m}(u+\Delta u, v)$. As long as we do this interpolation with a quadratic or a polynomial of higher degree, it does not seem to influence the secondorder convergence of the method. Finally, we can extract the Regge-Wheeler-Zerilli functions from the line $B C$ in Fig. 1 as they propagate toward future null infinity.

\section{Waveforms and radiated energy momentum}

As we mentioned at the end of the previous section, it is easy to find the Regge-Wheeler-Zerilli functions from the exterior of our computational grid; this is useful, because these functions are directly related to the gravitational waveform $h$, asymptotically. For radii much larger than the reduced gravitational wavelength, $r \gg \lambda_{\mathrm{GW}} /(2 \pi)$, one has that

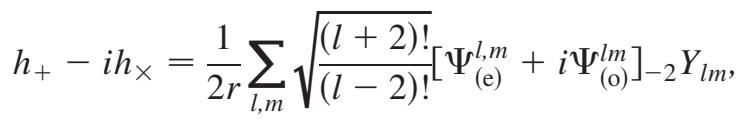

where ${ }_{-2} Y_{l, m}$ is a spin-weighted spherical harmonic. The above comes from Eq. (84) of Ruiz et al. [29], which also contains a discussion about the spin-weighted harmonics in an Appendix. One can then substitute this into the usual expressions for the energy and momentum radiated by gravitational waves,

$$
\begin{aligned}
\frac{d E}{d t} & =\lim _{r \rightarrow \infty} \frac{r^{2}}{16 \pi} \oint\left|\dot{h}_{+}-i \dot{h}_{\times}\right|^{2} d \Omega, \\
\frac{d P_{i}}{d t} & =\lim _{r \rightarrow \infty} \frac{r^{2}}{16 \pi} \oint n_{i}\left|\dot{h}_{+}-i \dot{h}_{\times}\right|^{2} d \Omega,
\end{aligned}
$$

(where $n_{i}$ is a unit vector, a dot represents a time derivative, and $d \Omega$ is the volume element on a 2 -sphere). A lengthy, but straightforward calculation done by Ruiz et al. [29] shows that

$$
\frac{d E}{d t}=\frac{1}{64 \pi} \sum_{l . m} \frac{(l+2) !}{(l-2) !}\left(\left|\dot{\Psi}_{(\mathrm{e})}^{l, m}\right|^{2}+\left|\dot{\Psi}_{(\mathrm{o})}^{l, m}\right|^{2}\right)
$$

[their Eq. (91)]. For the components of the momentum we are interested in (in the $x y$-plane) combining their Eqs. (86)-(88) and (93) and using their definition $P_{+}=$ $P_{x}+i P_{y}$ gives

$$
\begin{aligned}
\frac{d P_{+}}{d t}= & -\frac{1}{16 \pi} \sum_{l \cdot m} \frac{(l+2) !}{(l-2) !}\left[i a_{l, m} \dot{\Psi}_{(\mathrm{e})}^{l, m} \dot{\bar{\Psi}}_{(\mathrm{o})}^{l, m+1}\right. \\
& \left.+b_{l+1, m+1}\left(\dot{\Psi}_{(\mathrm{e})}^{l, m} \dot{\bar{\Psi}}_{(\mathrm{e})}^{l+1, m+1}+\dot{\Psi}_{(\mathrm{o})}^{l, m} \dot{\bar{\Psi}}_{(\mathrm{o})}^{l+1, m+1}\right)\right] .
\end{aligned}
$$

The bar denotes complex conjugation. The coefficients $a_{l . m}$ and $b_{l, m}$ are given by their Eqs. $(41,42)$, which we reproduce here 


$$
\begin{gathered}
a_{l . m}=\frac{\sqrt{(l-m)(l+m+1)}}{l(l+1)} \\
b_{l, m}=\frac{1}{2 l} \sqrt{\frac{(l-2)(l+2)(l+m)(l+m-1)}{(2 l-1)(2 l+1)}} .
\end{gathered}
$$

With the framework now in place, we are prepared to make a comparison with numerical relativity.

\section{HEAD-ON COLLISION OF SPINNING BLACK HOLES WITH TRANSVERSE, ANTIPARALLEL SPINS}

In this section, we discuss the specific example of a head-on collision of equal-mass black holes with transverse, antiparallel spins. We will specialize the general framework presented in Sec. II to the current configuration in the first subsection and then make the comparison with numerical relativity in the second.

\section{A. The Hybrid model for the head-on collision}

We will mimic the configuration used in the numericalrelativity simulation for ease of comparison. We thus choose our two black holes, labeled by $A$ and $B$, to have masses $M_{A}=M_{B}=M / 2$, to start with initial separation $X_{A}=A(0) / 2=-X_{B} \quad[A(0)=7.8 M \quad$ in the numerical simulations and $\left.Y_{A}=Y_{B}=Z_{A}=Z_{B}=0\right]$ and to have their spins along $\pm Z$ axis, respectively, (so that $S_{A}^{Z}=$ $0.5 M_{A}^{2}$ and $S_{B}^{Z}=-0.5 M_{B}^{2}$ and all other components of the spins are zero). Though they initially fall from rest, as in the numerical simulation, we will denote their speeds by $V_{A}$ and $V_{B}$.

\section{Even-Parity perturbations}

As we argued in Sec. II, the even-parity perturbation will only rely upon the Newtonian potential, which has the familiar form,

$$
U_{N}=\frac{M_{A}}{R_{A}}+\frac{M_{B}}{R_{B}} .
$$

Here $R_{A}$ and $R_{B}$ denote the distance from the centers of black holes $A$ and $B$ in the PN coordinates. We then expand the Newtonian potential, $U_{N}$, into multipoles, keeping only the monopole and quadrupole pieces (the dipole piece vanishes),

$$
\begin{aligned}
U_{N} & =U_{N}^{(0)}+U_{N}^{(2)} \\
& =\frac{M}{R}+\frac{M A(t)^{2}}{4 R^{3}} \sqrt{\frac{3 \pi}{10}}\left[Y_{2,-2}-\sqrt{\frac{2}{3}} Y_{2,0}+Y_{2,2}\right] .
\end{aligned}
$$

$Y_{l, m}$ are the usual scalar spherical harmonics. One can then see that the nonzero coefficients of the spherical harmonics are

$$
U_{N}^{2, \pm 2}=\sqrt{\frac{3 \pi}{10}} \frac{M A(t)^{2}}{4 R^{3}}=-\sqrt{\frac{3}{2}} U_{N}^{2,0} .
$$

After applying the transformation of the PN and Schwarzschild radial coordinates, $R=r-M$, [and similarly $A(t)=a(t)-2 M]$ one finds that

$$
U_{N}^{2, \pm 2}=\sqrt{\frac{3 \pi}{10}} \frac{M(a(t)-2 M)^{2}}{4(r-M)^{3}}=-\sqrt{\frac{3}{2}} U_{N}^{2,0} .
$$

One can then substitute this into Eq. (24) to find the Zerilli function,

$$
\Psi_{(\mathrm{e})}^{2, \pm 2}=\sqrt{\frac{3 \pi}{10}} \frac{M a(t)^{2}}{2 r^{2}}\left(1-\frac{7 M}{6 r}\right)=-\sqrt{\frac{3}{2}} \Psi_{(\mathrm{e})}^{2,0} .
$$

We have only kept terms to linear order in $M / r$ in this calculation, because we only use Newtonian physics to calculate the gravitational potential. At the boundary of $r_{s}(t)=a(t) / 2$, the perturbation is constant at leading order, and varying only at higher orders.

$$
\begin{aligned}
& \left.\Psi_{(\mathrm{e})}^{2, \pm 2}\right|_{\text {shell }}=\sqrt{\frac{6 \pi}{5}} M\left(1-\frac{7 M}{3 a(t)}\right), \\
& \left.\Psi_{(\mathrm{e})}^{2,0}\right|_{\text {shell }}=-\sqrt{\frac{4 \pi}{5}} M\left(1-\frac{7 M}{3 a(t)}\right) .
\end{aligned}
$$

\section{Odd-Parity perturbations}

The calculation with the gravitomagnetic potential is slightly more difficult, because it involves additional gauge transformations. The gravitomagnetic potential is given by

$$
w_{i}=\frac{\epsilon_{i j k} S^{j} N_{A}^{k}}{2 R_{A}^{2}}+\frac{M_{A} V_{A}^{i}}{4 R_{A}}+\frac{\epsilon_{i j k} S^{j} N_{B}^{k}}{2 R_{B}^{2}}+\frac{M_{B} V_{B}^{i}}{4 R_{B}} .
$$

These results appear, for example, in Eq. (6.1d) of [25]. The new symbols $N_{A}^{k}$ and $N_{B}^{k}$ represent unit vectors pointing from the centers of the two black holes in the PN coordinates, and $V_{A}^{i}$ and $V_{B}^{i}$ are the velocities of the two black holes. Expanding the gravitomagnetic potential to leading order in $A(t)$ and simplifying the trigonometric portions of the equations below, we see that

$$
\begin{gathered}
w_{x}=\frac{M V A(t) \sin \theta \cos \varphi}{2 R^{2}}-\frac{3 A(t) S \sin ^{2} \theta \sin 2 \varphi}{4 R^{3}}, \\
w_{y}=-\frac{A(t) S\left(1+3 \cos 2 \theta-6 \sin ^{2} \theta \cos 2 \varphi\right)}{8 R^{3}}, \\
w_{z}=0 .
\end{gathered}
$$

The variables $S$ and $V$ are just the magnitudes of the spin and velocity of each black hole, respectively. For this equal-mass collision, the spins have the same magnitude, and the velocities of the holes do, as well. We must then convert the gravitomagnetic potential into spherical-polar coordinates, 


$$
\begin{gathered}
w_{R}=\frac{M V A(t) \sin ^{2} \theta \cos ^{2} \varphi}{2 R^{2}}-\frac{A(t) S \sin \theta \sin \varphi}{2 R^{3}}, \\
w_{\theta}=\frac{M V A(t) \sin 2 \theta \cos ^{2} \varphi}{4 R}-\frac{A(t) S \cos \theta \sin \varphi}{2 R^{2}}, \\
w_{\varphi}=-\frac{M V A(t) \sin ^{2} \theta \sin 2 \varphi}{4 R} \\
+\frac{A(t) S \cos \varphi(5 \sin \theta-3 \sin 3 \theta)}{8 R^{2}} .
\end{gathered}
$$

There is a dipole term in the component $w_{R}$ of the gravitomagnetic potential, and this term will not evolve according to the Regge-Wheeler-Zerilli equation. One can remove it via the small gauge transformation to the metric,

$$
\hat{h}_{\alpha \beta}=h_{\alpha \beta}-\xi_{\alpha \mid \beta}-\xi_{\beta \mid \alpha},
$$

where the bar refers to a covariant derivative with respect to the background metric (in this case flat space). Recall that the metric components, $h_{t i}$, are related to the gravitomagnetic potential, $w_{i}$, by $h_{t i}=-4 w_{i}$. If we make a gauge transformation where the only nonzero component of $\xi_{\mu}$ is

$$
\xi_{t}=\frac{2 M A(t) V \sin ^{2} \theta \cos ^{2} \varphi}{R}-\frac{A(t) S \sin \theta \sin \varphi}{R^{2}},
$$

this has several important effects. For one, it eliminates $\hat{h}_{t r}$, and it introduces a term,

$$
-\xi_{t \mid t}=-\frac{4 M \sin ^{2} \theta \cos ^{2} \varphi}{R}\left(V^{2}-\frac{M A(t)}{2 R^{2}}\right),
$$

into $h_{t t}$. This term, however, is of 1PN order, and, since we are considering only the leading Newtonian physics, we will drop it. Then, it turns the remaining perturbation into the sum of odd- and even-parity quadrupole perturbations. Letting $b=\theta, \varphi$, one has that

$$
\begin{aligned}
\hat{h}_{t b}= & -\frac{A(t) S}{R^{2}} \sqrt{\frac{6 \pi}{5}}\left(X_{b}^{2,1}-X_{b}^{2,-1}\right), \\
& -\sqrt{\frac{2 \pi}{15}} \frac{4 M A(t) V}{R}\left(Y_{b}^{2,2}-\sqrt{\frac{2}{3}} Y_{b}^{2,0}+Y_{b}^{2,-2}\right) .
\end{aligned}
$$

If one were to include the even-parity, vector-harmonic term in the Zerilli function, one would need to take its time derivative. This means it enters as a next-to-leading-order effect, and we can ignore that term in our leading-order treatment. Thus, the relevant perturbation of the gravitomagnetic potential is

$$
w_{(\mathrm{o})}^{2,1}=\sqrt{\frac{6 \pi}{5}} \frac{A(t) S}{4 R^{2}}=-w_{(\mathrm{o})}^{2,-1} .
$$

Finally, we make the transformation to the Schwarzschild radial coordinate, $R=r-M$ [and similarly for $A(t)=$ $a(t)-2 M]$, to find that

$$
w_{(\mathrm{o})}^{2,1}=\sqrt{\frac{6 \pi}{5}} \frac{(a(t)-2 M) S}{4(r-M)^{2}}=-w_{(\mathrm{o})}^{2,-1} .
$$

We can then find the Regge-Wheeler function from Eq. (25) which is

$$
\Psi_{(\mathrm{o})}^{2,1}=-\sqrt{\frac{6 \pi}{5}} \frac{2 a(t) S}{r^{2}}=-\Psi_{(\mathrm{o})}^{2,-1} .
$$

As before, we keep only terms linear in $M / r$. At the boundary, the odd-parity perturbation is

$$
\left.\Psi_{(\mathrm{o})}^{2,1}\right|_{\text {shell }}=-\frac{8 S}{a(t)} \sqrt{\frac{6 \pi}{5}}=-\left.\Psi_{(\mathrm{o})}^{2,-1}\right|_{\text {shell }} \cdot
$$

\section{Energy and momentum fluxes}

Finally, because we only have quadrupole perturbations, the expressions for the energy and momentum fluxes greatly simplify. The energy flux, for the $l=2$ modes (taking into account that the $m= \pm 1$ are equal and the $m= \pm 2$ modes are equal, as well) becomes

$$
\dot{E}=\frac{3}{8 \pi}\left[\frac{8}{3}\left(\dot{\Psi}_{(\mathrm{e})}^{2,2}\right)^{2}+2\left(\dot{\Psi}_{(\mathrm{o})}^{2,1}\right)^{2}\right],
$$

and the momentum flux is given by

$$
\dot{P}_{y}=\frac{1}{\pi} \dot{\Psi}_{(\mathrm{e})}^{2,2} \dot{\Psi}_{(\mathrm{o})}^{2,1} .
$$

We have also used the fact that $\Psi_{(\mathrm{e})}^{2,0}=\sqrt{2 / 3} \Psi_{(\mathrm{e})}^{2, \pm 2}$ in this head-on collision.

\section{B. Comparison with numerical relativity}

In this section, we compare the results of our head-on collision of spinning black holes (with transverse, antiparallel spins) with the equivalent results from a numericalrelativity simulation (see the paper by Lovelace et al. [32] for a complete description of the simulation). Although the paper by Lovelace et al. dealt mostly with using the Landau-Lifshitz pseudotensor to define a gauge-dependent 4-momentum and an effective velocity to help develop intuitive understanding of black-hole collisions, they also investigated the gauge-invariant gravitational waveforms and radiated energy momentum (calculated from the gravitational waves at large radii). We will not attempt to study any of these Landau-Lifshitz quantities in this work, and, instead, we will just look into the gauge-invariant radiated quantities. Specifically, for our comparison, we focus on the waveforms (the $l=2$ modes of the gravitational waves) and the radiated energy and momentum.

In Figs. 4 and 5, we compare, respectively, the evenparity perturbation $\Psi_{\mathrm{even}}^{(2,2)}$ and the odd-parity perturbation $\Psi_{\text {odd }}^{(2,1)}$ from our method with the equivalent quantities from the numerical simulation $\mathrm{S} 1$ featured in Lovelace et al. (Since the $l=2, m=-2,-1,0$ components are related 


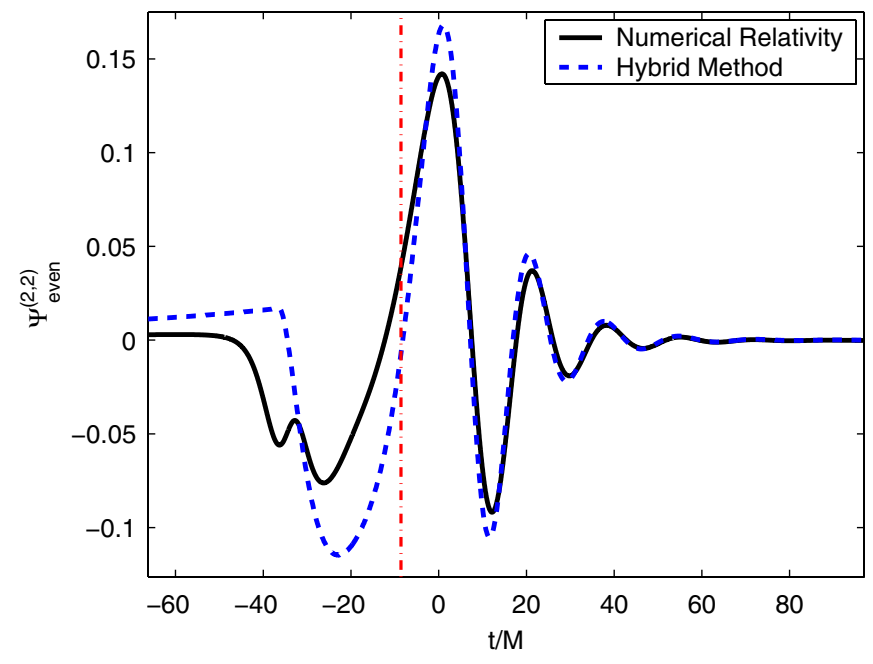

FIG. 4 (color online). The blue (dark gray) dashed curve is $\Psi_{\text {even }}^{2,2}$ from our hybrid method, whereas the black solid curve is the same quantity in full numerical relativity. The red (light gray) dashed vertical line corresponds to the retarded time at which the shell in the hybrid method reaches the light ring of the final black hole, $r=3 M$. We shift the numerical-relativity waveform so that the peaks of the numerical and hybrid $\Psi_{\text {even }}^{2,2}$ waveforms align.

by constant multiples to the above perturbations, we do not show them.) In these figures, we also include a vertical, dashed red line that indicates the retarded time at which the shell crossed the light ring of the final black hole $r=3 \mathrm{M}$, in the hybrid method. This is the peak of the effective potential, and due to the low-frequency opacity of this potential, much of the influence of the boundary data is hidden within the potential after this time (and the wave-

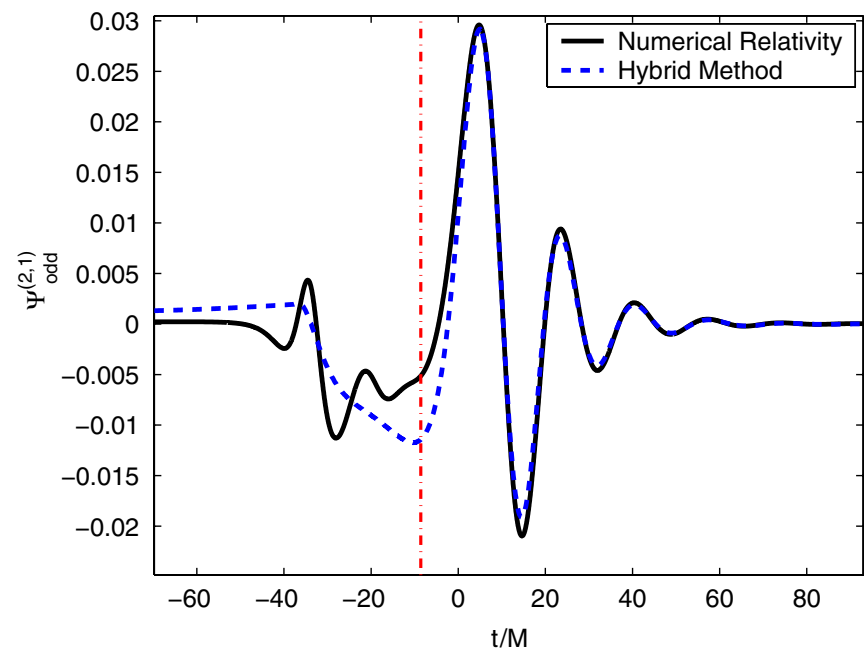

FIG. 5 (color online). The blue (dark gray) dashed curve is $\Psi_{\text {odd }}^{(2,1)}$ from our hybrid method, whereas the black solid curve is the same quantity in full numerical relativity. The red (light gray) dashed vertical line corresponds to the retarded time at which the shell in the hybrid method reaches the light ring of the final black hole, $r=3 M$. We shift the numerical-relativity waveform so that the peaks of the numerical and hybrid $\Psi_{\text {even }}^{2,2}$ waveforms align. form is due mostly to the quasinormal modes of the final black hole). Before this time the match is not exact (as a result of junk radiation in the numerical simulation and the difference between the time coordinates), but the Newtonian order perturbations do quite a good job of exciting quasinormal modes of a reasonable amplitude.

The even- and odd-parity waveforms in the hybrid method are the pieces of $\Psi_{\text {even }}^{(2,2)}$ and $\Psi_{\text {odd }}^{(2,1)}$ restricted to the outer boundary of the characteristic grid, labeled by $\mathrm{BC}$ in Fig. 1. We found these perturbations through the procedure described in Sec. II B, applied to the specific binary parameters described in Sec. III A. For the numerical-relativity waveforms, we chose to present them in terms of the even- and odd-parity perturbation functions $\Psi_{\text {even }}^{(2,2)}$ and $\Psi_{\text {odd }}^{(2,1)}$, as well. To find these perturbation functions from the numerical simulations, we first twice integrated the Weyl scalar $\Psi_{4}$ with respect to time to get the waveforms $h_{+}$and $h_{\times}$(since $-\Psi_{4}=\ddot{h}_{+}-i \ddot{h}_{\times}$, at large radii, where a dot denotes a time derivative). One can relate them to the gravitational waveforms $h_{+}$and $h_{\times}$ by Eq. (31), at large $r$. In the case of the $l=2$ perturbations shown here, $r h_{+}^{(2,2)}=\sqrt{6} \Psi_{\text {even }}^{(2,2)}$ and $r h_{\times}^{(2,1)}=\sqrt{6} \Psi_{\text {odd }}^{(2,1)}$. We compared the $h_{+}$and $h_{\times}$found directly from the numerical simulation through extraction of the ReggeWheeler and Zerilli functions from metric coefficients in the numerical code [33], and the two procedures gave essentially identical results.

In order to make the comparison between our hybrid method and the full numerical-relativity waveforms, we must shift the numerical waveforms by a constant. Specifically, we choose this constant so that the peaks of the exact and approximate waveforms of $\Psi_{\mathrm{even}}^{(2,2)}$ match (at a time that we set to be $t=0$ ). We add this constant shift in time, because there is no clear relationship between the coordinate time at which the waveform in our code begins and the same coordinate time in the numerical-relativity simulation. Trying to find a relationship between these times is complicated by the fact that the hybrid method evolves on a characteristic grid, whereas the numerical-relativity simulation solves an initial-value problem in a gauge that changes as the black holes move together. Nevertheless, because both the numerical and the hybrid method use asymptotically flat coordinates, at large radii, the time coordinates move at the same rate. This, in turn, means that it is only necessary to shift the time coordinates rather than rescaling them. As an interesting aside, Owen [34] found that this agreement between the time coordinates in the numerical simulation and perturbation theory appears to extend even into the near zone, when he observed that multipole moments of the horizon oscillate at the quasinormal mode frequencies of the black hole. See the end of Sec. 3 of that paper for a discussion of why that might be the case.

We also compute the momentum flux, and we show the accumulation of the velocity of the final black hole in Fig. 6, for both our method and the full numerical-relativity 


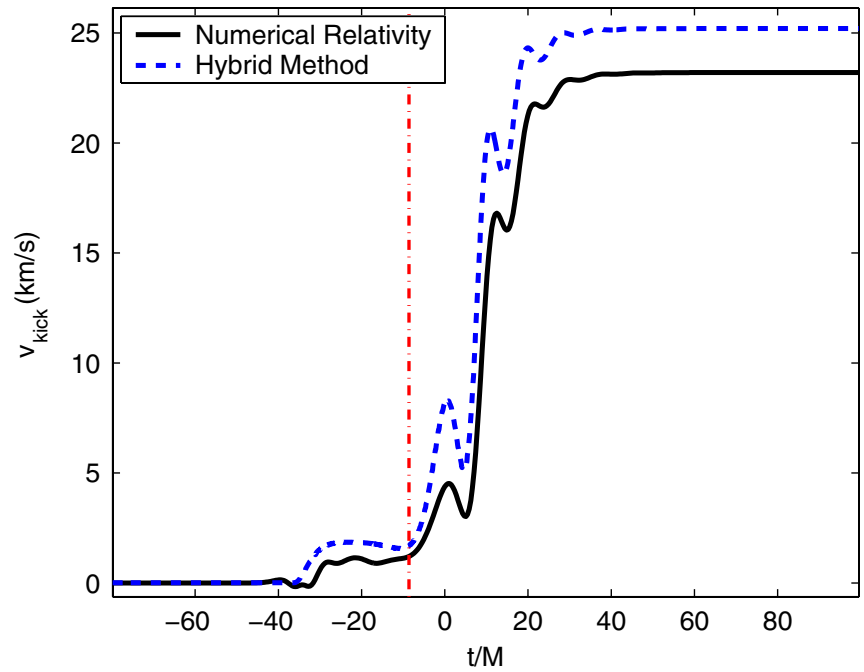

FIG. 6 (color online). The blue (dark gray) dashed curve is the velocity of the final black hole as a function of time (inferred from the gravitational radiation) from our hybrid method, using only the $l=2$ modes of the wave. The black solid curve is the equivalent quantity in numerical relativity, computed from the full Weyl scalar, $\psi_{4}$. The red (light gray) dashed vertical line corresponds to the retarded time at which the shell in the hybrid method reaches the light ring of the final black hole, $r=3 \mathrm{M}$. As before, the numerical-relativity velocity is shifted so that the peaks of the numerical and hybrid $\Psi_{\text {even }}^{2,2}$ waveforms align.

simulation. For our hybrid method, we use just the $l=2$ modes of the waveform to compute the momentum flux, $\dot{P}_{y}$, our Eq. (61). We then find the velocity of the final black hole as a function of time by computing

$$
v_{y}(t)=-\frac{1}{M} \int_{t_{0}}^{t} \dot{P}_{y}\left(t^{\prime}\right) d t^{\prime},
$$

where we introduce an extra minus sign to account for the fact that the black hole's velocity is opposite that of the momentum carried by the gravitational waves. For the numerical waveform, we show the equivalent velocity computed from the full Weyl scalar, $\Psi_{4}$. For the numerical simulations, one typically computes

$$
\dot{P}_{y}=\lim _{r \rightarrow \infty} \frac{r^{2}}{16 \pi} \oint \sin \theta \sin \phi\left|\int_{-\infty}^{t} \Psi_{4} d t^{\prime}\right|^{2} d \Omega
$$

where $\Psi_{4}$ is the Weyl scalar extrapolated to infinity, and $d \Omega$ is the surface-area element on a unit sphere. This expression appears in a variety of sources [see, for example, Eq. (29) of the paper by Ruiz et al. [29]]. We then can compute the velocity of the final black hole in the numerical simulations through Eq. (62), as we did for the hybrid method. Again, we perform the same timeshifting procedure as we did with the waveforms. The kick we find is remarkably close; $22 \mathrm{~km} / \mathrm{s}$ for the numerical simulation and $25 \mathrm{~km} / \mathrm{s}$ for our hybrid method.
The radiated energy does not agree quite as well due to the fact that the even-parity perturbation is somewhat larger than the equivalent numerical quantity (and it is the dominant contribution to the energy flux). Nevertheless, the results agree within a factor of 2; the numerical simulation shows that roughly $0.057 \%$ of the initial mass is radiated, whereas our hybrid method predicts approximately $0.096 \%$ of the initial mass escapes in gravitational waves. This is an improvement over some of the earlier, first-order, close-limit calculations at larger separations (see, for example, Fig. 1 of [35], where more than $1 \%$ of the initial mass is radiated for equivalent initial separations of the black holes).

We are not aware of any equivalent calculations of radiated energy for head-on collisions within the EOB formalism. While there have been recent 3PN calculations of the energy flux for a head-on plunge of black holes by Mishra and Iyer [36], in PN-based calculations such as the EOB approach, one must always stop the calculation at some radial separation of the binary. For example, in Fig. 2 of Mishra and Iyer's work, they stop the calculation at a point at which only roughly a tenth of the total energy that will be radiated in the head-on collision has escaped. This poses a small problem for EOB approaches, because as described in the Introduction to this paper, Sec. I, one must choose a point at which to stop the EOB inspiral and plunge waveform and match it to a set of quasinormal modes to obtain a full waveform. For inspiraling black holes, there is a natural point to do this: when the frequency of the inspiral-plunge dynamics approaches the quasinormal mode frequencies of the final black hole that will be formed. For a head-on collision, however, there is no analogous frequency at which one can match. We will, therefore, reserve any comparisons between our method and that of EOB for future work, when we extend our method to inspiraling, black-hole binaries.

\section{THE THREE STAGES OF BLACK-HOLE MERGERS}

In addition to producing reasonably accurate full waveforms, our approach also provides a possible interpretation of the infall, merger, and ringdown stages of a binaryblack-hole merger. As shown in Fig. 1, before the shell reaches point $e$ and enters the strong-field region [the red (dark gray) area, in which the $l=2$, even-parity, effective potential exceeds $1 / 3$ of its peak value], the majority of the retarded waveform propagates along the light cone (the socalled direct part). The direct part overwhelms the waves that scatter off the background curvature, because, far away from the source, the curvature is small. This part of the waveform corresponds to the inspiral or infall phase.

In the strong-field region, however, there is strong curvature (the black-hole effective potential in our model). While some fraction of the waves will pass directly through, as the shell enters this region at point $e$, waves 
that scatter off the effective potential (and thereby propagate within the light cone) become more significant. These waves often are called tail waves. Although PN waveforms do include the tail part, the fact that the higher-order PN terms that contain the tail dominate over the lower-order terms [24] does not bode well for the ability of the PN series to easily capture this effect. Nevertheless, we are able to associate this mixture of direct and tail portions in the waveform to merger. In our model, this stretch of the waveform is related to the retarded times when the shell is passing through the strong-field region of space-time (points $e, f$, and $g$ in the diagram).

Finally, after the shell passes through the potential, the details of the perturbation no longer become important, as was found by Price in his stellar collapse model. Because waves do not efficiently propagate through the barrier, the gravitational waveform associated with points $g$ through $D$ should arise from before and while the shell passes through the effective potential (not after). This last piece is that of ringdown.

There is one subtlety to note about our interpretation of ringdown that might arise if the final black hole is a Kerr black hole. Mino and Brink [37] and Zimmerman and Chen [38] showed that mergers that lead to a Kerr black hole can emit waves at integer multiples of the horizon frequency that decay at a rate proportional to the surface gravity. These modes come from a calculation in the nearhorizon limit of a Kerr black hole, and from the vantage point of observers far away, these waves would appear to be coming from the horizon. These modes have a sufficiently high frequency that they could penetrate the effective potential of a Kerr black hole and contribute to the ringdown portion of the gravitational wave. Nevertheless, if we expand our description of the ringdown phase to include these horizon modes, our interpretation holds more or less as described above.

\section{CONCLUSION}

In this paper, we show, by examining the head-on collisions of spinning black holes, that a combination of PN and BHP theories gives a gravitational waveform that matches well with that of full numerical-relativity simulations. We were able to do this not by applying the approximation methods to distinct times in the evolution of the system, but by choosing regions of space in which the two methods work and finding that the waveform from black-holebinary collisions can be protected from lack of conver- gence in these approximations. Specifically, our method lumps all monopole pieces of a PN black-hole-binary space-time into the Schwarzschild metric and treats the higher multipoles as perturbations of that Schwarzschild that evolve via a wave equation. Moreover, since PN and BHP theories describe the waveform, this suggests that much of the nonlinear dynamics appearing in the gravitational waves of a head-on black-hole-binary merger can be well approximated by linear perturbations of the Schwarzschild solution.

Our approach certainly cannot replace full numerical simulations. For one, we must test its validity for different kinds of coalescence by comparison with fully nonlinear numerical results. Nevertheless, we are hopeful that our method maybe be useful for gaining further understanding of the space-time of black-hole-binary mergers and for producing templates of gravitational waveforms for data analysis. To move towards these goals, we would need to make several modifications to our method (whose implementation we leave for future work). Most of these changes revolve around finding a way to treat inspirals of blackholes binaries within our method. The most necessary addition would be finding a way to consistently treat radiation reaction within the formalism. This feature is essential for capturing the correct inspiral and plunge dynamics. Also important for describing realistic physics of the ringdown would be to analyze the problem in a Kerr background. Each of these problems requires significant work, so we leave them for future studies.

\section{ACKNOWLEDGMENTS}

We thank Geoffrey Lovelace and Uli Sperhake for supplying waveforms and energy-momentum fluxes from their numerical simulations; we thank Lee Lindblom, Mark Scheel, and Béla Szilágyi for advice on solving wave equations with characteristic methods. We thank Drew Keppel for his input in discussions during the early stage of this work, and we thank Kip S. Thorne and Yasushi Mino for discussing related aspects of black-hole physics with us. This work has been supported by NSF Grants No. PHY0601459, No. PHY-0653653, and CAREER Grant No. PHY-0956189, by the David and Barbara Groce startup funds at the California Institute of Technology, and by the Brinson Foundation. D. N.'s research was supported by the David and Barbara Groce Graduate Research Assistantship at the California Institute of Technology.
[1] F. Pretorius, Phys. Rev. Lett. 95, 121101 (2005).

[2] M. Campanelli, C. O. Lousto, P. Marronetti, and Y. Zlochower, Phys. Rev. Lett. 96, 111101 (2006).
[3] J. G. Baker, J. Centrella, D.-I. Choi, M. Koppitz, and J. van Meter, Phys. Rev. Lett. 96, 111102 (2006).

[4] M. A. Scheel et al., Phys. Rev. D 79, 024003 (2009). 
[5] L. Rezzolla, R. P. Macedo, and J. L. Jaramillo, Phys. Rev. Lett. 104, 221101 (2010).

[6] R. H. Price and J. Pullin, Phys. Rev. Lett. 72, 3297 (1994).

[7] G. Khanna et al., Phys. Rev. Lett. 83, 3581 (1999).

[8] C. W. Misner, Phys. Rev. 118, 1110 (1960).

[9] R. W. Lindquist, J. Math. Phys. (N.Y.) 4, 938 (1963).

[10] D. R. Brill and R.W. Lindquist, Phys. Rev. 131, 471 (1963).

[11] J. M. Bowen and J. W. York, Phys. Rev. D 21, 2047 (1980).

[12] J. Baker, M. Campanelli, and C. O. Lousto, Phys. Rev. D 65, 044001 (2002).

[13] C. F. Sopuerta, N. Yunes, and P. Laguna, Phys. Rev. D 74, 124010 (2006).

[14] C. F. Sopuerta, N. Yunes, and P. Laguna, Astrophys. J. 656, L9 (2007).

[15] A. Le Tiec and L. Blanchet, Classical Quantum Gravity 27, 045008 (2010).

[16] A. Le Tiec, L. Blanchet, and C. Will, Classical Quantum Gravity 27, 012001 (2010).

[17] N. K. Johnson-McDaniel, N. Yunes, W. Tichy, and B. J. Owen, Phys. Rev. D 80, 124039 (2009).

[18] A. Buonanno et al., Phys. Rev. D 79, 124028 (2009).

[19] T. Damour and A. Nagar, Phys. Rev. D 79, 081503(R) (2009).

[20] A. Buonanno and T. Damour, Phys. Rev. D 62, 064015 (2000).
[21] R. H. Price, Phys. Rev. D 5, 2419 (1972).

[22] L. Blanchet and G. Faye, Phys. Rev. D 63, 062005 (2001).

[23] A. Garat and R. H. Price, Phys. Rev. D 61, 044006 (2000).

[24] E. Racine, A. Buonanno, and L. Kidder, Phys. Rev. D 80, 044010 (2009).

[25] J. D. Kaplan, D. A. Nichols, and K. S. Thorne, Phys. Rev. D 80, 124014 (2009).

[26] T. Regge and J. A. Wheeler, Phys. Rev. 108, 1063 (1957).

[27] F. J. Zerilli, Phys. Rev. D 2, 2141 (1970).

[28] V. Moncrief, Ann. Phys. (N.Y.) 88, 323 (1974).

[29] M. Ruiz, M. Alcubierre, D. Núñez, and R. Takahashi, Gen. Relativ. Gravit. 40, 1705 (2008).

[30] H. Stephani, General Relativity: An Introduction to the Theory of the Gravitational Field (Cambridge University Press, Cambridge, 1990), 2nd ed., Sec. 22.2.

[31] C. Gundlach, R. H. Price, and J. Pullin, Phys. Rev. D 49, 883 (1994).

[32] G. Lovelace et al., Phys. Rev. D 82, 064031 (2010).

[33] O. Rinne, L. T. Buchman, M. A. Scheel, and H. P. Pfeiffer, Classical Quantum Gravity 26, 075009 (2009).

[34] R. Owen, Phys. Rev. D 80, 084012 (2009).

[35] R.J. Gleiser, C.O. Nicasio, R.H. Price, and J. Pullin, Phys. Rev. Lett. 77, 4483 (1996).

[36] C. K. Mishra and B. R. Iyer, arXiv:1008.4009.

[37] Y. Mino and J. Brink, Phys. Rev. D 78, 124015 (2008).

[38] A. Zimmerman and Y. Chen (unpublished). 